\title{
Improvement of Electronic Compass Accuracy Based on Magnetometer and Accelerometer Calibration
}

\author{
M. ŠIPOŠ* , J. ROHÁČ, P. NOVÁČEK \\ Czech Technical University in Prague, Faculty of Electrical Engineering, Department of Measurement \\ Technická 2, 166 27, Prague, Czech Republic

\begin{abstract}
This paper describes the process used for an electronic compass compensation according to accelerometer based tilt evaluation. Tilt angles have to be estimated first for sensed magnetic vector components to be aligned and horizontal components evaluated. Therefore the precision of accelerometer based tilt angles plays a key role in this whole process as well as the magnetometer characteristics. Hence accelerometers plus magnetometers have to be calibrated to improve the accuracy of a tilt and an azimuth angle evaluation. The calibration uses Thin-Shell method to determine sensor error models. Both the effect of calibration and precision of estimated error models have been observed and are presented. The electronic compass consisted of tri-axial magnetometer and tri-axial accelerometer contained in the Inertial Measurement Unit ADIS16405 from Analog Devices manufacturer.
\end{abstract}

PACS: 85.75.Ss, 91.10.-v, 06.20.fb, 91.25.-r, 07.07.Df

\section{Introduction}

Since 1500 years ago, the mechanical compasses have been used for an azimuth determination and a guidance using Earth magnetic field. Due to the technology development and improvement, current electronic compasses (ECs) have much better parameters which are, of course, influenced by sensor type applied. The most simple low accuracy compasses use Hall sensors. In contrast, more accurate ones use Anisotropic Magneto Resistors (AMR) and the most accurate compasses use the fluxgate sensors [1]. The final accuracy of EC depends not only on used magnetic sensors, but also on tilt sensors, which have to be utilized to mathematically align magnetic sensors (compasses with tilt compensation) into the local navigation frame. Characteristics of tilt sensors also affect the EC accuracy, and therefore they have to be calibrated, which eliminates the sensors imperfections [2]. For lowcost sensors like MEMS (Micro-Electro-Mechanical System) based ones, manufacturers mostly perform only basic calibration and the rest is left on customers. Thus, for better accuracy the system needs to be recalibrated [3]. There exists a wide range of calibration procedures and techniques, e.g. the calibration using redundant heading information computed from rate gyroscopes [4] or the calibration procedure based on ellipsoid fitting problem which does not need heading reference information obtained from redundant sensors [5].

Nowadays, the ECs have become useful in a wide range of consumer applications such as mobile phones, PDAs, robot navigation, human head and hands tracking, attitude determination of inertial navigation systems used in aerospace engineering, etc. [1, 2, 6-8].

In this paper, the EC system and the tilt compensation is briefly described in section 2, the sensor error model (SEM) is further discussed in chapter 3 and Thin-Shell

\footnotetext{
* corresponding author; e-mail: siposmar@fel.cvut.cz
}

calibration method is mentioned in section 4 . A measurement setup and a measured unit are briefly introduced in section 5. The most important results are summarized in section 6 .

\section{Electronic compass}

The simplest electronic compass (EC) can be constructed using only a dual-axis magnetometer. This type of EC can measure accurate only azimuth (yaw angle) in horizontal plane. The resulting azimuth $\psi$ can be computed using simple eq. (1):

$$
\psi=\arctan \left(f_{y} / f_{x}\right)-D
$$

where $f_{x}, f_{y}$ are horizontal magnetic field components measured in sensor (body) frame, and $D$ is a magnetic declination [2].

Although this type of compasses is very simple and easy to manufacture, a main disadvantage of this EC construction is in the obligation to place the sensor accurately into horizontal plane. If it cannot be ensured, the errors are not negligible as was proved by Vcelak in [2]. Generally, it is not possible to ensure this condition providing horizontal mounting of magnetic sensor, so the electronic compass has to be equipped with tilt compensation functionality. The compass with tilt compensation (Fig. 1) usually consists of tri-axial magnetometer and tilt sensor, which can be formed by tri-axial accelerometer [9] or an electronic inclinometer commonly used in Honeywell compasses.

The EC uses magnetometer platform mathematically aligned to the horizontal plane using pitch and roll angles defined by (2) and (3). The azimuth can be then computed using (4).

$$
\begin{aligned}
& \theta=\arctan \left(-a^{b y} / \sqrt{\left(a^{b x}\right)^{2}+\left(a^{b z}\right)^{2}}\right), \\
& \varphi=\arctan \left(a^{b x} /\left(-a^{b z}\right)\right),
\end{aligned}
$$




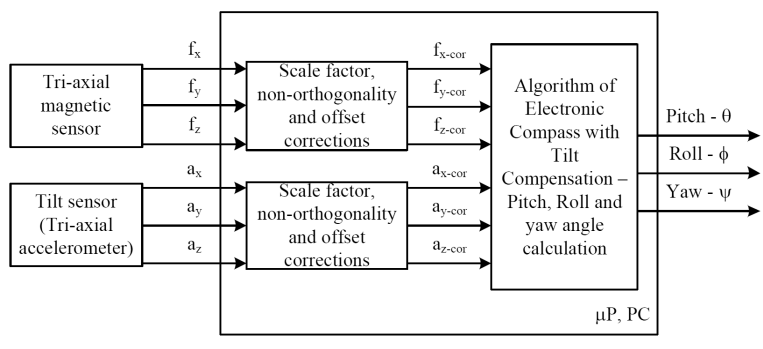

Fig. 1. The block scheme of an EC with a tilt compensation and the compensation of sensor imperfections formed in the sensor error model (5).

$$
\begin{aligned}
& \psi= \\
& \arctan \frac{f_{y} \cos \varphi+f_{z} \sin \varphi}{f_{x} \cos \varphi+f_{y} \sin \varphi \sin \theta-f_{z} \cos \varphi \sin \theta}-D,
\end{aligned}
$$

where: $\theta, \varphi$ denote the pitch and roll angle; $a^{b x}, a^{b y}$, $a^{b z}$ are measured accelerations in the sensor body frame; $f_{x}, f_{y}, f_{z}$ represent magnetic field vector components measured in sensor frame [10].

\section{Sensor error model}

In the chapter 1 it was mentioned that the calibration is necessary to be performed for the elimination of the sensor imperfections. Generally, sensors have many error sources; nevertheless, our sensor error model (SEM) defined by (5) includes the main ones [11]. They correspond to scale factor deflections, axes misalignment described in our case by three non-orthogonality angles [11], and offsets for all three axes. The offset forms a stochastic time-invariant part of the bias; in contrast, a drift characterizes a time-variant part of the bias. Because the calibration process is commonly performed during shorttime period, the drift can be considered as zero [11].

$$
\begin{aligned}
& \boldsymbol{y}_{\boldsymbol{p}}=\boldsymbol{T}_{\boldsymbol{a}}^{\boldsymbol{p}} \boldsymbol{S} \boldsymbol{F}_{\boldsymbol{a}}\left(\boldsymbol{y}_{\boldsymbol{m}}-\boldsymbol{b}_{\boldsymbol{a}}\right)\left(\begin{array}{ccc}
1 & 0 & 0 \\
\alpha_{y x} & 1 & 0 \\
\alpha_{z x} & \alpha_{z y} & 1
\end{array}\right) \\
& \times\left(\begin{array}{ccc}
S F_{a x} & 0 & 0 \\
0 & S F_{a y} & 0 \\
0 & 0 & S F_{a z}
\end{array}\right)\left[\left(\begin{array}{l}
y_{m x} \\
y_{m y} \\
y_{m z}
\end{array}\right)-\left(\begin{array}{c}
b_{a x} \\
b_{a y} \\
b_{a z}
\end{array}\right)\right]
\end{aligned}
$$

where $\boldsymbol{y}_{p}$ represents the compensated vector of either measured acceleration in the case of accelerometers or magnetic field vector in case of magnetometers and is defined in the orthogonal platform frame; $\boldsymbol{T}_{\boldsymbol{a}}^{\boldsymbol{p}}$ denotes the matrix providing the transformation from the non-orthogonal frame to the orthogonal one with nondiagonal terms $\alpha_{y x}, \alpha_{z x}, \alpha_{z y}$ that correspond to the axes misalignment; $S F_{a}$ represents a scale factor matrix; $\boldsymbol{b}_{\boldsymbol{a}}=\left[b_{a x}, b_{a y}, b_{a z}\right]^{T}$ is the vector of offsets; $\boldsymbol{y}_{\boldsymbol{m}}=$ $\left[y_{m x}, y_{m y}, y_{m z}\right]^{T}$ denotes the vector of measured acceleration/magnetic field vector. The SEM and its derivation are described in more detail in [12].

\section{Calibration procedure}

There already exist several calibration procedures for tri-axial sensors using different principles, e.g. the method using an ellipsoidal-fitting procedure [5, 13], a calibration procedure which uses a robotic arm [14] or a procedure with the usage of $3 \mathrm{D}$ optical tracking system that measures the position coordinates of markers attached to a measurement unit [15].

In our case, we used the thin-shell (TS) calibration method. A fundamental principle of the proposed method is based on the fact that the magnitude of measured quantity $|y|$ (gravity acceleration, magnetic field vector) should be always equal to the constant value when static conditions are ensured and also equal to the square root of the sum of squared vector components (6):

$$
y_{x}^{2}+y_{y}^{2}+y_{z}^{2}=|y|^{2},
$$

where $y_{i}$ denotes sensed quantity in direction of $i$ axis and $|y|$ is the magnitude of measured quantity. In the case of the gravity vector, it is ideally equal to $\mathrm{lg}$ and in the case of the magnetic field vector $|F|$ it is equal to $0.48125 G$ for the location (area) where the measurements were taken. The value of Earth magnetic field vector was calculated using International Geomagnetic Reference Field model (IGRF 11) which depends on the date of measurement, GPS position, and the altitude [16].

For the calibration purposes, according to [11], 36 positions are recommended to measure, 3 times 12 positions along $x, y, z$ axis. The advantage of the method is that the precise knowledge of position orientations is not required. It is only recommended to provide at least 3 positions per each quadrant and each axis. After the measurements are taken, the Thin-Shell algorithm can be applied on the measured data. The TS algorithm is based on a lineaer minimum mean square error principle minimizing the standard deviation $\sigma$ defined by (7), which is calculated from compensated vector component estimates and the known number of measurements.

$$
\sigma=\sqrt{\frac{\sum_{i=1}^{m}\left(\widehat{y}_{x i}^{2}+\widehat{y}_{y i}^{2}+\widehat{y}_{z i}^{2}-|y|^{2}\right)^{2}}{m-1}},
$$

where $\widehat{y}_{x i}, \widehat{y}_{y i}, \widehat{y}_{z i}$ are estimations of compensated acceleration/magnetic field vector components and $|y|$ is the magnitude of the reference value corresponding to measured quantity. The more detailed description of this calibration method is presented in $[11,17]$.

In each iteration step the interval defines the minimum, maximum, and mean value of the parameter being searched for and these values are then used to update the SEM. Thus, 3 SEMs are obtained coresponding to min., max., and mean values of the given parameter. Based on the updated SEMs new estimates of compensated vector are determined for each position and used for $\sigma$ calculations. With respect to obtained 3 values of $\sigma$ the interval is halved to find the local minimum of a standard deviation according to Fig. 2. When $\sigma_{\text {mean }}$ reaches the smallest value, the interval is halved around $k_{\text {mean }}$, where " $k$ " rep- 
resents the parameter being searched for. Unlikely, when other $\sigma$ reaches the smallest value and $\sigma_{\text {mean }}$ is the second, the new interval is defined between $\mathrm{k}$, whose $\sigma$ was the smallest and $k_{\text {mean }}$. For instance, when $k_{\text {min }}$ has the smallest $\sigma$, the $k_{\text {mean }}$ becomes $k_{\text {max }}$ for another iteration step and the new $k_{\text {mean }}$ is calculated as the average of the new $k_{\max }$ and previous $k_{\min }$. The same principle can be applied for the other case [11].

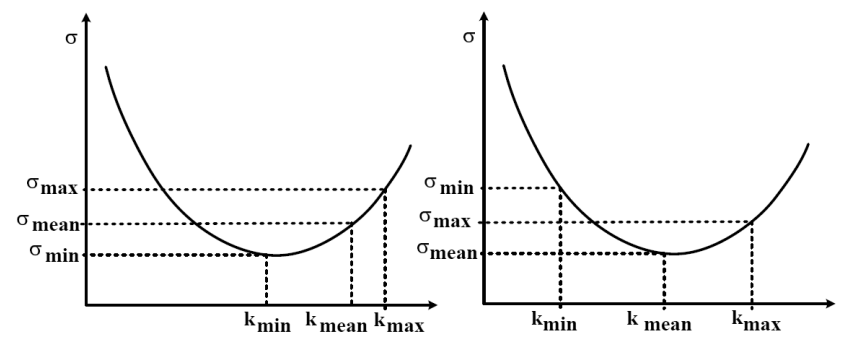

Fig. 2. Criterions for halving the interval, in which the estimated parameters are searched for [11].

\section{Measurement setup}

In our case the measurement setup was built up by the inertial measurement unit (IMU) ADIS16405 [18] (Analog Devices) and the non-magnetic theodolite T1c (Meopta Prague, Czech Republic). The IMU was used to evaluate the EC algorithm with tilt compensation and to prove the improvement of applied calibration procedure. The IMU (Fig. 3) contains the tri-axial magnetometer (MAG), tri-axial accelerometer (ACC), and triaxial angular rate sensor (ARS). The measurements were performed in the area with minimal magnetic field disturbances in the local time from 18:00 to 19:00 CET when the variations of magnetic field are minimal. For the evaluation of EC accuracy, the IMU was mounted on the non-magnetic theodolite, see Fig. 3, which was used as a reference with an average error $4.17 \times 10^{-3} \mathrm{deg}$.

In all performed experiments we used for calibration purposes and a final EC evaluation the average of 100 ACC and MAG samples taken in each position under static conditions as a value we consequently calculated with. A main reason for the usage of average values was the elimination of a noise influence.

\section{Results}

\subsection{Calibration of Magnetometer and Accelerometer of IMU ADIS16405}

From the output data provided by IMU ADIS16405 we used only information from the magnetometer (MAG) and the accelerometer (ACC). After the data had been preprocessed, the calibration was performed using the Thin-Shell algorithm to estimate three misalignment angles (non-orthogonality angles), three scale factor corrections, and three biases, all formed in SEM (5). The
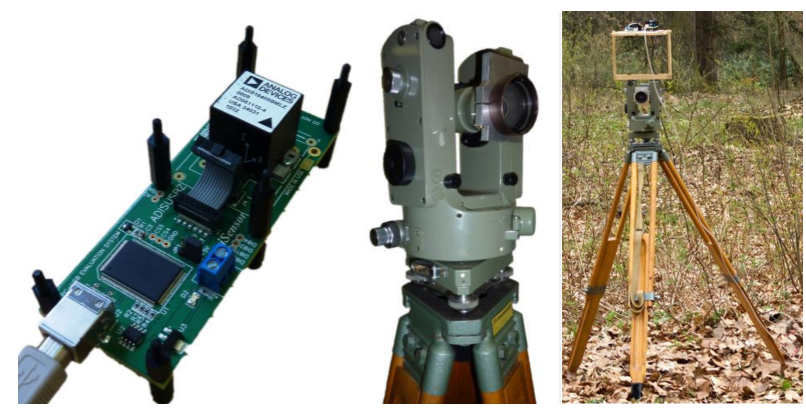

Fig. 3. The Inertial measurement unit ADIS16405 (on the left); theodolite T1c (in the middle); the whole measurement setup (on the right).

parameters of MAG and ACC SEMs are listed in Table I. The deviation between the measured and the ideal vector of applied quantity (corresponds to the magnetic field vector for MAG and to the gravity vector for ACC) is shown in Fig. 4 and Fig. 5. In contrast with the chapter 4 , in which 36 positions are recommended for a correct calibration, we used only 21 positions in the case of MAG. The measurement took shorter time and thus we minimized the risk of potential magnetic field variations. In [11] it was proven that 21 positions is a sufficient number without a final accuracy decrease. For ACC calibration, the 36 positions were measured as was recommended.

TABLE I

Sensor error models obtained using Thin-Shell algorithm for magnetometer (MAG) and accelerometer (ACC) of IMU ADIS16405 (Superscript 1 denotes RMSE before calibration and 2 after calibration)

\begin{tabular}{c|c|c}
\hline \hline Parameter & MAG & ACC \\
\hline$\alpha_{x y}[\mathrm{deg}]$ & 0.1355 & -0.0230 \\
$\alpha_{z x}[\mathrm{deg}]$ & -0.6628 & 0.0351 \\
$\alpha_{z y}[\mathrm{deg}]$ & -0.0818 & -0.1639 \\
$\mathrm{SF}_{x}[-]$ & 1.0049 & 0.9996 \\
$\mathrm{SF}_{y}[-]$ & 1.0050 & 1.0019 \\
$\mathrm{SF}_{z}[-]$ & 1.0004 & 0.9983 \\
$\mathrm{~b}_{x}$ & $-0.71 \mathrm{mG}$ & $-13.54 \mathrm{mg}$ \\
$\mathrm{b}_{y}$ & $-0.83 \mathrm{mG}$ & $-6.71 \mathrm{mg}$ \\
$\mathrm{b}_{z}$ & $0.23 \mathrm{mG}$ & $-4.02 \mathrm{mg}$ \\
$\mathrm{RMSE}^{1}$ & $1.9 \mathrm{mG}$ & $9.5 \mathrm{mg}$ \\
$\mathrm{RMSE}^{2}$ & $0.4 \mathrm{mG}$ & $2.5 \mathrm{mg}$
\end{tabular}

\subsection{Influence of $M A G$ and $A C C$ Calibration to Electronic Compass Accuracy}

Finally, we analyzed in previous chapter performed calibration from the final accuracy of realized electronic compass (EC) point of view. We performed four measurements at all. In each measurement the EC was differently tilted in two directions to set values of $0 \mathrm{deg}$ and $20 \mathrm{deg}$ in various combinations. Then, the azimuth was 


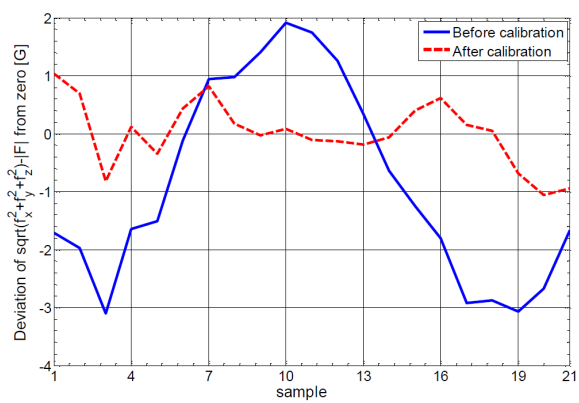

Fig. 4. The dependence of deviations of measured magnetic field vector before and after calibration MAG of ADIS16405 - 21 evaluated positions.

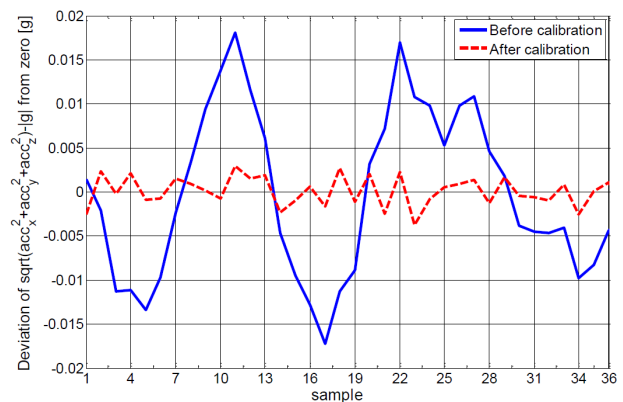

Fig. 5. The dependence of deviations of measured accelerations before and after calibration - ACC of ADIS16405 - 36 evaluated positions.

changing with the step of $22.5 \mathrm{deg}$ and a tilt compensation observed as well as the effect of MAG and ACC calibration on the azimuth accuracy. As a criterion for the azimuth accuracy evaluation the RMSEs were computed and the final values with and without calibration (applied SEM) summarized, see Table II. The table provides the final RMSEs depending on set tilts in two direction (pitch and roll angles). In all four data sets, the application of evaluated SEMs led to the improvement of the final EC accuracy.

TABLE II

Final accuracy of yaw angle estimation with and without applied ACC and MAG $\mathrm{SEM} ; \theta$ - pitch, $\varphi$ - roll, $\psi$ - yaw

\begin{tabular}{c|c|c|c}
\hline \hline$\theta$ [deg] & $\varphi[\mathrm{deg}]$ & without & with \\
\cline { 3 - 4 } & & calibration & $\Delta \psi_{\text {RMSE }}[\mathrm{deg}]$ \\
\hline 0 & 0 & 1.663 & 0.534 \\
0 & 20 & 1.270 & 0.462 \\
20 & 0 & 2.012 & 0.567 \\
20 & 20 & 1.303 & 0.563
\end{tabular}

\section{Conclusion}

This paper deals with an electronic compass (EC) algorithm and procedures needed for its correct functionality.
The EC performance generally depends on used tri-axial magnetometer (MAG) and its parameters as well as on parameters of an aligning system. In our case we used tri-axial accelerometer (ACC) for this purpose. To improve EC performance we applied a calibration procedure Thin-Shell to estimate sensor error models of MAG and ACC. The methods were shortly introduced; nevertheless, a main focus was pointed to present experimental results. We performed the calibration of MAG, which approximately five-times improved its accuracy and in the case of ACC the accuracy was four-times improved. Although the calibration procedure recommended 36 positions to use, we measured data only 21 in the case of MAG which was in accordance to [11]. In contrast, for the ACC calibration we kept 36 positions as was recommended. We analyzed the influence of MAG and ACC calibration on the final EC accuracy by analyzing the differences between the evaluated azimuth and the reference angle obtained from our reference system formed by theodolite T1c. The evaluated azimuth reflected estimated SEMs' parameters, which were: three scale-factors corrections, three non-orthogonality angles, and three offsets. In all tested experiments the application of MAG and ACC SEMs led to improvement of final EC accuracy as was presented.

\section{Acknowledgement}

This research has been partially supported by Czech Science Foundation project 102/09/H082, partially by the research program No. MSM6840770015 "Research of Methods and Systems for Measurement of Physical Quantities and Measured Data Processing" of the CTU in Prague sponsored by the Ministry of Education, Youth and Sports of the Czech Republic and partially by Grant Agency of the Czech Technical University in Prague grant No. SGS10/288/OHK3/3T/13.

\section{References}

[1] R. Racz, Ch. Schott, S. Huber, Proceedings of the IEEE sensors 2004, Vienna, Austria 1-3, 1446, (2005).

[2] J. Vcelak, V. Petrucha, P. Kaspar, Sensor Letters 5, 279, (2005).

[3] Z. Syed, P. Aggarwal, C. Goodall, X. Niu, N. ElSheimy, Measurement Science \& Technology 18, 1897, (2007).

[4] B. Hoff, R. Azuma, IEEE and ACM International Symposium on Augmented Reality, Munich, Germany, 159, (2000).

[5] X. Hu, Y. Liu, Y. Wang, Y. Hu, D. Yan, 4th IEEE/ACM International Symposium on Mixed and Augmented Reality 2005, Vienna, Austria, 182-183, (2005).

[6] V.Y. Skvortzov, HK. Lee, S.W. Bang, YB. Lee, Proceedings of the 2007 IEEE International Conference on Robotics and Automation 1-10, 2963, (2007).

[7] M. Reinstein, Przeglad Elektrotechniczny 87, 255, (2011). 
[8] M. Reinstein, M. Sipos, J. Rohac, Przeglad Elektrotechniczny 85, 114, (2009).

[9] J. Včelák, P. Ripka, J. Kubík, A. Platil, P. Kašpar, Sensors and Actuators A: Physical 123-124, 122, (2005).

[10] M. Soták, Przegląd Elektrotechniczny 86, 247, (2010).

[11] M. Sipos, P. Paces, J. Rohac, P. Novacek, IEEE Sensors Journal, (2011), accepted for publication.

[12] I. Skog, P. Händel, XVII IMEKO World Congress, Rio de Janeiro, (2006).

[13] S. Bonnet, C. Bassompierre, C. Godin, S. Lesecq, A. Barraud, Sensors and Actuators A: Physical 156, 302, (2009).

[14] E.L. Renk, M. Rizzo, W. Collins, F. Lee, D.S. Bernstein, IEEE Control Systems Magazine, 25, 86, (2005).

[15] A. Kim, MF. Golnaraghi, Plans 2004: Position location and Navigation Symposium, Monterey, CA, 26, (2004).
[16] NOAA's Geophysical Data Center - Geomagnetic Online Calculator. Available: http://www.ngdc.noaa.gov/ geomagmodels/ IGRFWMM.jsp [Accessed: 09-Sep-2011].

[17] M. Soták, M. Sopata, R. Bréda, J. Roháč, L. Váci, Navigation System Integration, Košice: Robert Breda, Kosice, the Slovak Republic (2006).

[18] ADIS16405 High Precision Tri-Axis Gyroscope, Accelerometer, Magnetometer, Inertial Sensors, Sensors, Analog Devices. Available: http://www.analog.com/en/sensors/inertialsensors/adis16405/products/product.html [Accessed: 24-Apr-2011]. 